PAPER

\title{
Do stroke patients with normal carotid arteries require TEE for exclusion of relevant aortic plaques?
}

\author{
A Harloff, M Handke, A Geibel, E Oehm, B Guschlbauer, M Olschewski, A Hetzel
}

J Neurol Neurosurg Psychiatry 2005;76:1654-1658. doi: 10.1136/jnnp.2005.065821

See end of article for authors' affiliations

.....................

Correspondence to: Dr A Harloff, Department of Neurology and Clinical Neurophysiology, AlbertLudwigs-Universität, Breisacher Straße 64, D79106 Freiburg (Germany); harloff@nz. ukl.uni-freiburg.de

Received 17 February 2005 Revised version received 4 May 2005

Accepted 6 May 2005

\begin{abstract}
Objectives: This study investigated (a) the hypothesis that stroke patients with aortic atheroma would show comparable atherosclerotic changes in the carotid arteries, which are easily accessible for ultrasound evaluation and $(b)$ the possibility of carotid duplex sonography as a replacement for transoesophageal echocardiography (TEE) for the exclusion or prediction of relevant aortic plaques.

Methods: In 301 consecutive patients (mean age 62 years) with acute cerebral ischaemia, two dimensional ultrasound measurements were taken of common carotid artery intima media thickness (IMT) and maximal plaque area (PA) and the local degree of internal carotid artery (ICA) stenosis were determined. Maximal aortic wall thickness (AWT) was assessed by TEE.

Results: An IMT $\leqslant 0.9 \mathrm{~mm}$ yielded a negative predictive value (NPV) of $95.8 \%$ for exclusion of aortic atheromas $\geqslant 4 \mathrm{~mm}$ and an NPV of $100 \%$ for the exclusion of aortic thrombi. However, positive predictive value of IMT $>0.9 \mathrm{~mm}$ was low $(29.6 \%$ ), increasing only slightly in the presence of carotid plaques (33\%). Incidence of aortic thrombi was significantly higher with $\geqslant 50 \%$ compared with $<50 \%$ ICA stenosis $111.3 \%$ $v 3.9 \%$, respectively). IMT and PA correlated moderately with AWT $(r=0.47, r=0.53$, respectively; $\mathrm{p}<0.001$ ). Systolic blood pressure, coronary heart disease and peripheral artery disease, increased IMT, and ICA stenosis $\geqslant 50 \%$ were independently related to AWT $\geqslant 4 \mathrm{~mm}$.

Conclusions: A high NPV of normal carotid ultrasound does not support routine TEE for the exclusion of complex aortic plaques as a high risk source of cerebral embolism. However, in patients with carotid atherosclerosis, particularly in those with ICA stenosis $\geqslant 50 \%$, TEE should be performed to exclude an additional high risk source for stroke.
\end{abstract}

ncreased carotid intima media thickness (IMT) indicates generalised atherosclerosis ${ }^{1-5}$ and is a strong predictor of ischaemic stroke. ${ }^{6-8}$ Similarly, aortic atherosclerosis is related to vascular risk factors and is also independently associated with cerebral ischaemia. ${ }^{9-12}$ The prevalence of both carotid artery disease and thick thoracic aortic plaques is comparable to that of atrial fibrillation in patients with ischaemic stroke. ${ }^{13}$ An association between carotid and aortic arteriosclerosis has been shown in smaller series ${ }^{14-16}$ and in a large epidemiological study. ${ }^{8}$ However, there has been no prospective evaluation of the exact relation using carotid duplex sonography and transoesophageal echocardiography (TEE) in a larger and unselected cohort of patients with ischaemic stroke.

We hypothesised that patients with aortic plaques would also show atherosclerotic changes in carotid arteries. These atherosclerotic changes are easier to evaluate with ultrasound and could therefore serve as an indicator for aortic plaques. Thus the aims of our study were:

- to determine the precise association of carotid and aortic atherosclerosis in a larger stroke population, taking into consideration both cardiovascular risk factors and laboratory markers of atherosclerosis

- to test the reliability of carotid duplex sonography as a replacement for TEE in the exclusion or prediction of clinically relevant aortic plaques.

\section{PATIENTS AND METHODS} Study population

We prospectively enrolled a total of 384 consecutive patients admitted to our stroke unit with acute cerebral ischaemia within the last 16 months. The local ethics committee approved the study and all patients gave written informed consent. TEE was not done in 56 patients for the following reasons: patient refused examination $(n=27)$, clinical contraindication $(n=19)$, and non-compliance or gastrointestinal tract obstruction $(\mathrm{n}=10)$. We excluded 12 patients because of unclear delineation of the IMT complex and 15 patients because the diagnosis on discharge was not transient ischaemic attack or ischaemic stroke. Hence the final sample consisted of a consecutive series of 301 patients.

The study protocol included a prospective evaluation of cardiovascular risk factors. All patients underwent cranial computed tomography or magnetic resonance imaging of the brain or both, duplex sonography of extracranial and intracranial arteries, transthoracic echocardiography, and TEE. Blood pressure was measured every 60 minutes on the stroke unit and average systolic and diastolic values were obtained from patients' charts as separate variables within the time interval of 24-48 hours after the qualifying event.

Blood samples were taken from the antecubital vein after overnight fasting. Cholesterol and triglycerides were measured using enzymatic methods and reagents (WAKO Chemicals; Neuss, Germany). Lipoproteins were measured with rapid electrophoresis (Helena Laboratories, Gateshead, UK). ${ }^{17}$ Glycosylated haemoglobin $\left(\mathrm{HbA}_{1 \mathrm{c}}\right)$ was measured with high performance liquid chromatography (glycohaemoglobin analyser; Tosoh Corp, Tokyo, Japan).

\footnotetext{
Abbreviations: AWT, aortic wall thickness; CCA, common carotid artery; DA, dimensions of atherosclerosis; $\mathrm{HbA}_{1 \mathrm{c}}$, glycosylated haemoglobin; ICA, internal carotid artery; IMT, intima media thickness; NPV, negative predictive value; PA, plaque area; PPV, positive predictive value; TEE, transoesophageal echocardiography
} 


\section{Evaluation of risk factor variables}

We considered patients to be exposed to a cardiovascular risk factor when one of the following criteria was satisfied:

- age $>60$ years

- male sex or $\geqslant 5$ years after menopause for women

- hypertension-that is, history of hypertension or blood pressure exceeding $160 \mathrm{~mm} \mathrm{Hg}$ systolic or $95 \mathrm{~mm} \mathrm{Hg}$ diastolic on two occasions in the hospital

- diabetes mellitus-that is, history of diabetes or fasting glucose exceeding $126 \mathrm{mg} / \mathrm{dl}$ or patient requiring glucose lowering therapy

- hypercholesterolaemia - that is, total plasma cholesterol of $>200 \mathrm{mg} / \mathrm{l}$ in the previous 12 months or documented hypercholesterolaemia requiring lipid lowering drug therapy

- smoking habit-that is, consuming $\geqslant 1$ cigarette daily

- documented previous ischaemic stroke/transient ischaemic attack

- coronary artery disease-that is, documented coronary artery stenosis, percutaneous transluminal coronary angioplasty or coronary artery bypass, myocardial infarction

- peripheral artery disease-that is, documented peripheral artery disease, percutaneous transluminal angioplasty, vascular surgery.

\section{Assessment of carotid atherosclerosis}

Two trained sonographers assessed the carotid arteries using duplex ultrasound scanning with a 4-7 MHz linear array scanner (ATL, HDI 3500; ATL Bothell, USA). With the patient in the supine position, a careful search was performed of all interfaces of up to 12 different sites in the transverse and sagittal planes (right and left, near and far walls, proximal and distal common carotid artery (CCA), and internal carotid artery (ICA)). When an optimal longitudinal image of the CCA was obtained, it was frozen and magnified at the time of the R wave on the electrocardiogram (ECG) and stored on SVHS videotape. Quantitative measurements of IMT were done offline. The readers who evaluated the ultrasound images on videotape were blinded to patient demographics and the results of the TEE examinations. IMT was measured twice on the far wall at a distance of $4 \mathrm{~cm}$ and $1 \mathrm{~cm}$ proximal to the bifurcation on both sides of the CCA on a plaque free site. For each patient the common carotid IMT was taken as the mean of eight IMT measurements ( $\left.\mathrm{IMT}_{\text {mean }}\right)$. Based on the $\mathrm{IMT}_{\text {mean, patients }}$ were classified into the following categories: I, $\leqslant 0.9 \mathrm{~mm}$; II, $>0.9 \mathrm{~mm}-1.05 \mathrm{~mm}$; II, $>1.05 \mathrm{~mm}-1.2 \mathrm{~mm}$.

Carotid plaque was defined as a focal circumscribed increase in intimal wall thickness. We measured plaque area (PA) in the most stenotic plaque of the bifurcation/ICA by multiplying plaque length by thickness. We estimated the dimensions of atherosclerotic change (DA) in the carotid arteries in a manner similar to that used previously by others ${ }^{18}$ : $\mathrm{IMT}_{\text {mean }} \times 80 \mathrm{~mm}(80 \mathrm{~mm}$ represents the $40 \mathrm{~mm}$ long segments of the left and right CCA proximal to the bifurcation)+PA of both ICA. In accordance with the European Carotid Surgery Trial (ECST) protocol $^{19}$ the degree of ICA stenosis was defined as the "local" degree of stenosis and categorised as either $<50 \%$ (on both ICAs) or $\geqslant 50 \%$ (left or right ICA). Corresponding values for "distal" degree of ICA stenosis are approximately $<30 \%$ and $\geqslant 30 \%$, respectively. ${ }^{20}$

\section{Assessment of aortic atherosclerosis}

TEE was performed with a $5 \mathrm{MHz}$ multiplane probe (ATL, HDI 3500) and recorded on S-VHS videotape. An experienced senior echocardiographer, blinded to patient demographics and carotid artery scanning data, reviewed the findings offline. The ascending aorta and the aortic arch including the outlet of the left subclavian artery were imaged in short axis and long axis views. Findings distal to the left subclavian artery (that is, in the descending aorta) were not considered in this study. Atherosclerosis was defined as irregular and circumscribed intimal thickening with increased echogenicity. ${ }^{14}$ Maximal aortic wall thickness (AWT) was measured and the presence of mobile thrombi documented. We classified the patients similar to a previous study': I, AWT $<1.0 \mathrm{~mm}$; II, plaques $1.0-3.9 \mathrm{~mm}$ thick; III, plaques $\geqslant 4.0 \mathrm{~mm}$ thick.

\section{Statistical analysis}

We used the SAS statistical package (version 6.12) for all statistical analyses. Data are presented as mean and standard deviation for continuous variables and as absolute and relative frequencies for categorical variables. We used Fisher's exact test to detect statistically significant relations between categorical variables, and for continuous variables, $t$ tests or Wilcoxon's tests were used as appropriate; all tests used were two sided. Correlations between selected variables were estimated by calculating Pearson's correlation coefficient. Carotid artery ultrasonographic data and corresponding TEE data were compiled into a $2 \times 2$ contingency table to allow calculation of predictive values. Multivariate analyses (analysis of covariance) were performed for continuous dependent variables (IMT and AWT) and multiple logistic regressions for dichotomised dependent variables (AWT $<4 \mathrm{~mm} v$ AWT $\geqslant 4 \mathrm{~mm}$ ). We set the level of significance at $5 \%$. We report nominal $\mathrm{p}$ values not adjusted for multiple comparisons.

\section{RESULTS \\ Baseline characteristics and cardiovascular risk factors}

The baseline characteristics of the 301 study participants are shown in table 1 . Complete data on blood lipids and $\mathrm{HbA}_{\mathrm{lc}}$ were available for 272 patients $(90.4 \%)$. Ultrasound measurements are given in table 2. Both IMT and AWT increased significantly with the number of cardiovascular risk factors $(\mathrm{p}<0.05)$.

\section{Carotid artery duplex ultrasonography data}

In the univariate regression analysis IMT correlated with increased systolic blood pressure and $\mathrm{HbA}_{\mathrm{lc}}(\mathrm{p}=0.001)$, LDL/ HDL cholesterol ratio $(\mathrm{p}=0.025)$ and decreased high density lipoprotein (HDL) cholesterol level $(\mathrm{p}<0.01)$, with age $>60$

Table 1 Baseline characteristics of the study population. Data are mean (SD)* or $\mathrm{n}(\%) \dagger$

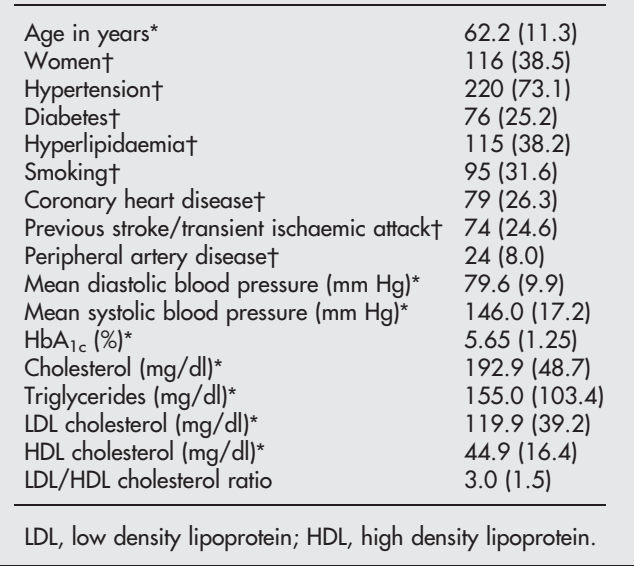


Table 2 Ultrasound measurements of intima media thickness (IMT) and aortic wall thickness (AWT). Data are mean $(\mathrm{SD})^{*}$ or $\mathrm{n}(\%) \dagger$

\begin{tabular}{|c|c|c|c|c|}
\hline \multirow[b]{2}{*}{ Characteristic } & \multirow[b]{2}{*}{ Value } & \multicolumn{2}{|l|}{ ICA stenosis } & \multirow[b]{2}{*}{ p valueł } \\
\hline & & $\begin{array}{l}<50 \% \\
(n=230)\end{array}$ & $\begin{array}{l}\geqslant 50 \% \\
(n=71)\end{array}$ & \\
\hline Mean IMT $(\mathrm{mm})^{*}$ & $1.02(0.21)$ & $0.99(0.20)$ & $1.09(0.22)$ & 0.0004 \\
\hline IMT $>0.75-0.9 \mathrm{~mm} \dagger$ & $95(31.6)$ & $84(36.5)$ & $11(15.5)$ & 0.0007 \\
\hline IMT $>0.9-1.05 \mathrm{~mm}$ & $101(33.6)$ & 77 (33.5) & $24(33.8)$ & \\
\hline IMT $>1.05-1.2 \mathrm{~mm}$ & 105 (34.9) & $69(30.0)$ & $36(50.7)$ & \\
\hline Mean AWT $(\mathrm{mm})^{*}$ & 2.95 (1.69) & $2.68(1.56)$ & $3.82(1.80)$ & $<0.0001$ \\
\hline $\mathrm{AWT}<1.0 \mathrm{~mm} \dagger$ & $19(6.3)$ & $19(8.3)$ & $0(0.0)$ & $<0.0001$ \\
\hline AWT 1.0-3.9 mm† & $217(72.1)$ & 177 (77.0) & $40(56.3)$ & \\
\hline $\mathrm{AWT} \geqslant 4.0 \mathrm{~mm} \dagger$ & $65(21.6)$ & $34(14.8)$ & $31(43.7)$ & \\
\hline Mobile thrombus $\dagger$ & $17(5.6)$ & $9(3.9)$ & $8(11.3)$ & 0.034 \\
\hline
\end{tabular}

years, hypertension and diabetes $(\mathrm{p}<0.001)$, male sex, and coronary and peripheral artery disease $(\mathrm{p}<0.05)$. Correlations of multivariate regression analysis are given in table 3 .

\section{Aortic findings}

Univariate regression analysis demonstrated correlation between AWT and increased systolic and diastolic blood pressure $(p=0.001), \mathrm{HbA}_{\mathrm{lc}}(\mathrm{p}<0.01), \mathrm{HDL}$ cholesterol (inversely) $(\mathrm{p}<0.05)$ and with age $>60$ years, hypertension, diabetes, smoking, previous brain ischaemia, coronary and peripheral artery disease $(\mathrm{p}<0.001)$. Correlations of multivariate regression analysis are shown in table 3.

\section{Correlation between carotid and aortic atherosclerosis}

The correlation between IMT and AWT was moderate (fig 1) and highest between the total dimension of carotid atherosclerosis and AWT (fig 2). The correlation of IMT and AWT with carotid plaque area was similar $(r=0.51$ and $r=0.53$, $\mathrm{p}<0.001)$.

Carotid stenosis and aortic findings

IMT and AWT were higher in patients with ICA stenosis $\geqslant 50 \%$ (table 2 ). Aortic plaques $\geqslant 4 \mathrm{~mm}$ occurred in $21.6 \%$ $(65 / 301)$ of the entire study population and they were almost three times more frequent with $\geqslant 50 \%$ ICA stenosis compared with $<50 \%$ stenosis $(43.7 \% \vee 14.8 \%)$. Mobile aortic thrombi were detected in $5.6 \%$ of patients (17/301); the AWT was $\geqslant 4 \mathrm{~mm}$ in $15(88.4 \%)$ and $<4 \mathrm{~mm}$ in only two of these patients. Similarly, in patients with $\geqslant 50 \%$ stenosis the incidence of aortic thrombi was almost three times higher than in patients with $<50 \%$ stenosis $(11.3 \% \vee 3.9 \%)$. There was no statistically significant difference between IMT and AWT, and the incidence of AWT $\geqslant 4 \mathrm{~mm}$ or aortic thrombi, in $50-79 \%$ compared with $\geqslant 80 \%$ ICA stenosis.

\section{Predictive values}

IMT $\leqslant 0.9 \mathrm{~mm}$, seen in 95 of our patients (31.6\%; table 2 ), almost excluded AWT $\geqslant 4.0 \mathrm{~mm}$ (negative predictive value (NPV) $95.8 \%(4 / 95))$. In the four false negatives, the AWT ranged from $4.1 \mathrm{~mm}$ to $4.5 \mathrm{~mm}$. The combination of IMT $\leqslant 0.9 \mathrm{~mm}$ and absence of carotid plaques, seen in 49 patients (16.3\%), increased the NPV to $100 \%$. On TEE none of the 95 patients with IMT $\leqslant 0.9 \mathrm{~mm}$ had aortic thrombi, but 2/95 $(2.1 \%)$ had left atrial thrombi. The positive predictive value (PPV) of IMT $>0.9 \mathrm{~mm}$ for the presence of aortic plaques
Table 3 Multivariate regression analysis* of carotid and aortic ultrasound measurements with demographic and clinical data

\begin{tabular}{llll}
\hline Characteristic & $\begin{array}{l}\text { IMT } \\
\text { (n=301) }\end{array}$ & $\begin{array}{l}\text { AWT } \\
(\mathbf{n}=301)\end{array}$ & $\begin{array}{l}\text { AWT } \geqslant 4 \mathbf{m m} \\
(\mathbf{n}=65)\end{array}$ \\
\hline Age $>60$ years & 0.002 & $<0.001$ & 0.079 \\
Diabetes & 0.005 & 0.002 & 0.116 \\
Coronary artery disease & 0.437 & 0.155 & 0.012 \\
Peripheral artery disease & $<0.001$ & $<0.001$ & 0.004 \\
Internal carotid artery stenosis & 0.094 & 0.001 & 0.001 \\
Systolic blood pressure & 0.035 & $<0.001$ & 0.028 \\
Decreased HDL cholesterol & 0.303 & 0.020 & 0.129 \\
LDL/HDL cholesterol ratio & 0.007 & 0.708 & 0.426 \\
\hline
\end{tabular}

*P values for the test of no effect of the corresponding characteristics on the dependent variables are given.

AWT, aortic wall thickness; HDL, high density lipoprotein; LDL, low

density lipoprotein; IMT, intima media thickness.

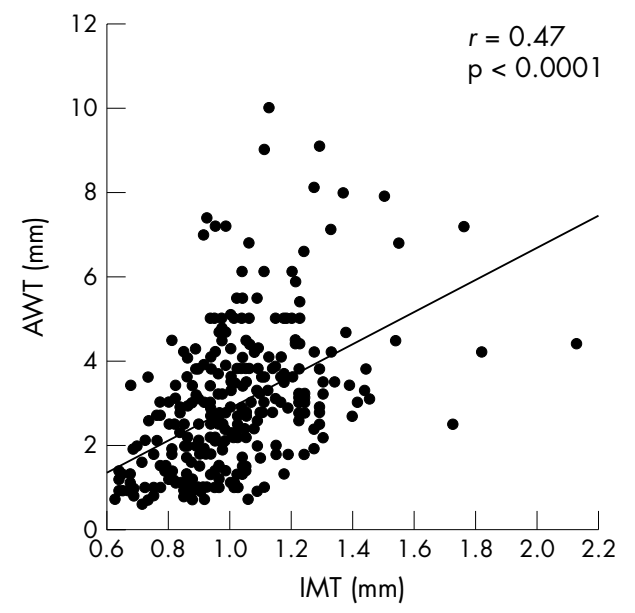

Figure 1 Correlation of intima media thickness (IMT) and aortic wall thickness (AWT).

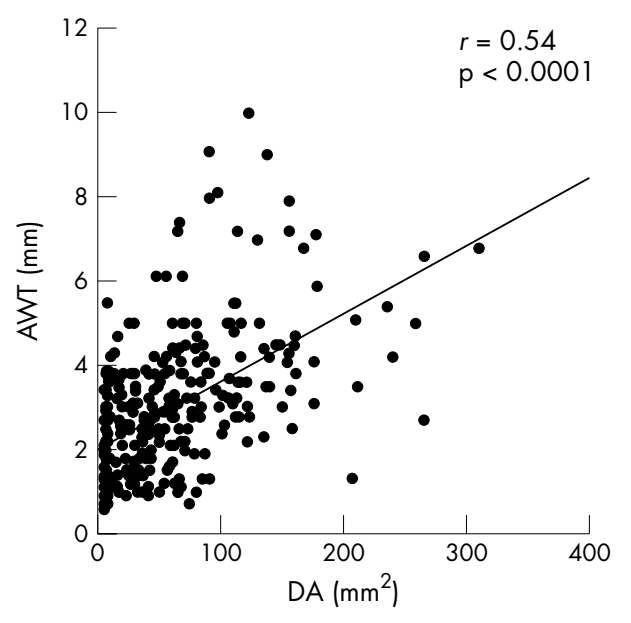

Figure 2 Correlation of dimensions of atherosclerosis (DA) and aortic wall thickness (AWT).

$\geqslant 4 \mathrm{~mm}$ was low $(29.6 \% ; 61 / 206)$, even when carotid plaques were taken into account $(33.0 \%, 59 / 179)$.

\section{DISCUSSION}

We present the first prospective investigation of a large cohort of consecutive patients with recent brain ischaemia 
evaluated by carotid ultrasound and TEE. We found a high NPV of normal carotid arteries for aortic thrombi and plaques $\geqslant 4 \mathrm{~mm}$ and a moderate correlation of atherosclerosis in the carotid arteries and in the aorta.

With regard to correlation between IMT, plaque area, and DA with AWT, our results are comparable with other studies. ${ }^{11}{ }^{14-16}$ Patients with ICA stenosis $\geqslant 50 \%$ had increased IMT and AWT, in particular, a three times higher incidence of superimposed aortic thrombi compared with those with lower grade ICA stenosis. However, the overall incidence of aortic thrombi was lower than has been described before, ${ }^{21}$ and the thrombi were found with all degrees of ICA stenosis ${ }^{913}$-not just with $\geqslant 80 \%{ }^{22}$ Therefore, TEE screening of the aorta seems to be of benefit in patients with advanced carotid atherosclerosis (that is, especially in ICA stenosis $\geqslant 50 \%$ ) particularly if localisation of brain infarction is at odds with the side of the stenosis. The detection of aortic thrombi on TEE may then change the secondary prevention strategy. However, the optimal therapy for aortic thrombi or plaques $\geqslant 4 \mathrm{~mm}$ has not yet been established: in three smaller studies warfarin was superior to aspirin in complex aortic plaques whereas in a larger retrospective analysis only statins showed a significant benefit. ${ }^{23}$ There are only case reports of successful ultima ratio treatment options such as aortic endarterectomy ${ }^{24}$ or aortic balloon thrombectomy. ${ }^{25}$ The ongoing Aortic arch Related Cerebral Hazard trial of 1500 patients comparing clopidogrel + aspirin versus warfarin may contribute substantial information towards the optimal management of aortic plaques.

Currently, the importance of TEE in the diagnostic workup of stroke patients lies in the detection of cardiac sources of cerebral embolism such as complex aortic plaques (that is, aortic thrombi or plaques $\geqslant 4 \mathrm{~mm}$ thick). TEE is also the current gold standard for the detection of left atrial or appendage thrombi, persistent foramen ovale, and atrial septal aneurysm. Therefore it plays a major role in guiding therapy and in the follow up of stroke patients. ${ }^{26}$ However, the high NPV of normal carotid IMT found in our study does not support routine TEE for the exclusion of complex aortic plaques as a high risk source of cerebral embolism in stroke patients. Screening of carotid arteries can be helpful for the exclusion of an atherosclerotic proximal embolic source when TEE or MRI is contraindicated, refused or not available. Moreover, when carotid arteries are normal a significant macroangiopathy can be broadly ruled out, and additional exclusion of cerebral microangiopathy can help to shorten the search for cardiovascular risk factors. The detection of two atrial thrombi in 95 patients with normal carotid arteries demonstrates that TEE should be considered on an individual basis to detect rare or unexpected sources of cerebral embolism. In contrast with Hollander et $a l^{8}$ our results suggest that there is a close correlation between carotid and aortic atherosclerosis, representing the same pathophysiological mechanism, providing reliable predictive values. This discrepancy between the results is most likely due to different study populations, examination of different segments of the aorta and the evaluation of aortic atheroma by $x$ ray versus ultrasound.

In agreement with others, ${ }^{15}{ }^{16}$ we found that the PPV of carotid ultrasound for relevant aortic atheromas is low. Shimizu et al ${ }^{16}$ showed a moderate association between carotid and aortic atherosclerosis and a significant increase in complex aortic plaque probability with increasing carotid IMT. Our results are comparable, especially for patients with $\geqslant 50 \%$ ICA stenosis. However, in Shimizu's study a cohort consisting of only 147 preselected patients was examined and their results thus might not be representative for all stroke causes. Unfortunately, exact PPVs or NPVs of carotid IMT for relevant aortic plaques have not been provided. Fasseas et $a l^{15}$ reported similar predictive values for complex aortic plaques in 64 stroke patients with severe carotid atherosclerosis (that is, IMT $\geqslant 2.0 \mathrm{~mm}$ ) whereas our results imply that even considerably less advanced carotid atherosclerosis helps in identifying patients with relevant aortic atherosclerosis. In 62 patients undergoing TEE for cardiological indications, ${ }^{14}$ the PPV of carotid plaques (that is, IMT $\geqslant 1.3 \mathrm{~mm}$ ) for aortic atherosclerosis (that is, luminal irregularity or wall thickness $>3 \mathrm{~mm}$, plaques with mobile thrombi or ulcerations) was $83 \%$, whereas NPV was only $63 \%$. These findings are in contrast with the studies cited above ${ }^{15} 16$ and with our results but this is probably because of the different definitions used by Kallikazaros $e t a l^{10}$ for carotid and aortic plaques. After the trials on aortic atherosclerosis, ${ }^{9}{ }^{13}$ it seems to be clinically more relevant to exclude plaques $\geqslant 4 \mathrm{~mm}$ thickness.

As shown previously, ${ }^{4} 91114$ 27-30 increased IMT and AWT in our patients correlated with classic cardiovascular risk factors. The independent prediction of aortic plaques $\geqslant 4 \mathrm{~mm}$ by indicators of peripheral atherosclerosis such as ICA stenosis, coronary, and peripheral artery disease emphasises the systemic nature of atherosclerosis.

\section{Limitations of the study}

Owing to the different ultrasound probe frequencies, measurement techniques, and patient populations, only limited comparisons can be made between studies on IMT. $^{5731}$ Measurement of mean IMT on the far wall of the CCA is a frequent technique, ${ }^{31}$ providing the clearest target for measurement and allowing use of an automatic detection system. ${ }^{5}$ The use of this technique and an ultrasound probe with higher resolution for both carotid and aortic ultrasound in the present study might have increased the accuracy of IMT and AWT measurements to a certain extent. However, at the time of the study it was not possible to do this in our stroke unit. Moreover, three dimensional ultrasound might have provided additional information in terms of plaque size and morphology in both territories.

\section{SUMMARY}

A normal carotid ultrasound offers a high NPV for the exclusion of relevant aortic atherosclerosis and makes TEE indispensable for this indication in stroke patients. Patients with $\geqslant 50 \%$ ICA stenosis have a high risk of severe aortic plaques or aortic thrombi. For a sophisticated planning of secondary prevention they should undergo TEE examination of the aorta.

\section{Authors' affiliations}

A Harloff, E Oehm, B Guschlbauer, A Hetzel, Department of Neurology and Clinical Neurophysiology, Albert-Ludwigs-Universität Freiburg,

\section{Germany}

M Handke, A Geibel, Department of Angiology and Cardiology, AlbertLudwigs-Universität Freiburg, Germany

M Olschewski, Institute of Medical Biometrics and Statistics, AlbertLudwigs-Universität Freiburg, Germany

Competing interests: none declared

\section{REFERENCES}

1 Grobbee D, Bots M. Carotid intima-media thickness as an indicator of generalized atherosclerosis. J Intern Med 1994;236:567-73.

2 Bots ML, Hoes AW, Koudstaal PJ, et al. Common carotid intima-media thickness and risk of stroke and myocardial infarction: The Rotterdam study. Circulation 1997;96:1432-7.

3 Allan PL, Mowbray PI, Lee AJ, et al. Relationship between carotid intimamedia thickness and symptomatic and asymptomatic peripheral arterial disease. The Edinburgh Artery Study. Stroke 1997;28:348-53.

4 Baldassarre D, Amato M, Bondioli A, et al. Carotid artery intima-media thickness measured by ultrasonography in normal clinical practice correlates well with atherosclerosis risk factors. Stroke 2000;31:2426-30.

5 O'Leary DH, Polak JF. Intima-media thickness: a tool for atherosclerosis imaging and event prediction. Am J Cardiol 2002;90:18L-21L. 
6 O'Leary DH, Polak JF, Kronmal RA, et al. Carotid-artery intima and media thickness as a risk factor for myocardial infarction and stroke in older adults. Cardiovascular Health Study Collaborative Research Group. N Engl J Med 1999;340:14-22

7 Touboul PJ, Elbaz A, Koller $\mathrm{C}$, et al Common carotid artery intima-media thickness and brain infarction: the Etude du Profil Genetique de l'Infarctus Cerebral (GENIC) case-control study. The GENIC Investigators. Circulation 2000;102:313-18.

8 Hollander M, Hak AE, Koudstaal PJ, et al. Comparison between measures of atherosclerosis and risk of stroke: The Rotterdam Study. Stroke 2003;34:2367-73.

9 The French Study of Aortic Plaques in Stroke Group. Atherosclerotic disease of the aortic arch as a risk factor for recurrent ischemic stroke. N Engl J Med 1996:334:1216-21.

10 Kallikazaros I, Tsioufis C, Sideris S, et al. Carotid artery disease as a marker for the presence of severe coronary artery disease in patients evaluated for chest pain. Stroke 1999;30:1002-7.

11 Jarvisalo MJ, Jartti L, Nanto-Salonen K, et al. Increased aortic intima-media thickness: a marker of preclinical atherosclerosis in high-risk children. Circulation 2001;104:2943-7.

12 Fujimoto S, Yasaka M, Otsubo R, et al. Aortic arch atherosclerotic lesions and the recurrence of ischemic stroke. Stroke 2004;35:1426-9.

13 Amarenco $\mathbf{P}$, Cohen A, Tzourio $C$, et al. Atherosclerotic disease of the aortic arch and the risk of ischemic stroke. N Engl J Med 1994;331:1474-9.

14 Kallikazaros IE, Tsioufis CP, Stefanadis Cl, et al. Closed relation between carotid and ascending aortic atherosclerosis in cardiac patients. Circulation 2000;102(suppl):III263-III268.

15 Fasseas $\mathbf{P}$, Brilakis ES, Leybishkis B, et al. Association of carotid artery intimamedia thickness with complex aortic atherosclerosis in patients with recent stroke. Angiology 2002;53:185-9.

16 Shimizu Y, Kitagawa K, Nagai Y, et al. Carotid atherosclerosis as a risk factor for complex aortic lesions in patients with ischemic cerebrovascular disease. Circ J 2003:67:597-600.

17 Aufenanger J, Haux P, Kattermann R. Improved method for enzymic determination of cholesterol in lipoproteins separated by electrophoresis on thin layer agarose gels. J Clin Chem Clin Biochem 1989;27:807-13.

18 Spence JD, Eliasziw M, DiCicco M, et al. Carotid plaque area: a tool for targeting and evaluating vascular preventive therapy. Stroke 2002;33:2916-22.
19 MRC European Carotid Surgery Trial: interim results for symptomatic patients with severe (70-99\%) or with mild (0-29\%) carotid stenosis. European Carotid Surgery Trialists' Collaborative Group. Lancet 1991;337:1235-43.

20 Von Reutern GM, von Büdingen HJ. Ultrasound diagnosis of cerebrovascular disease. Stuttgart: Thieme, 1993.

21 Arko FR, Fritcher S, Mettauer M, et al. Mobile atheroma of the aortic arch and the risk of carotid artery disease. Am J Surg 1999;178:206-8

22 Demopoulos LA, Tunick PA, Bernstein NE, et al. Protruding atheromas of the aortic arch in symptomatic patients with carotid artery disease. Am Heart J 1995; 129:40-4.

23 Tunick PA, Nayar AC, Goodkin GM, et al. Effect of treatment on the incidence of stroke and other emboli in 519 patients with severe thoracic aortic plaque. Am J Cardiol 2002;90:1320-5.

24 Geha AS, El-Zein C, Massad MG, et al. Surgery for aortic arch thrombosis. Thorac Cardiovasc Surg 2004;52:187-90.

25 Schneiderman J, Feinberg MS, Schwammenthal E, et al. Protruding aortic arch thrombus: treatment with minimally invasive surgical approach. J Vasc Surg 2004;40:1083-8.

26 Reynolds HR, Tunick PA, Kronzon I. Role of transesophageal echocardiography in the evaluation of patients with stroke. Curr Opin Cardiol 2003;18:340-5.

27 Smilde TJ, van Wissen S, Wollersheim $\mathrm{H}$, et al. Effect of aggressive versus conventional lipid lowering on atherosclerosis progression in familial hypercholesterolaemia (ASAP): a prospective, randomised, double-blind trial. Lancet 2001;357:577-81.

28 Suurkula M, Agewall S, Fagerberg B, et al. Ultrasound evaluation of atherosclerotic manifestations in the carotid artery in high-risk hypertensive patients. Risk Intervention Study (RIS) Group. Arterioscler Thromb 1994; 14:1297-304.

29 Davila-Roman VG, Murphy SF, Nickerson NJ, et al. Atherosclerosis of the ascending aorta is an independent predictor of long-term neurologic events and mortality. J Am Coll Cardiol 1999;33:1308-16.

30 Van der Meer IM, Iglesias del Sol A, Hak AE, et al. Risk factors for progression of atherosclerosis measured at multiple sites in the arterial tree: the Rotterdam Study. Stroke, 2003;34;2374-9..

31 Aminbakhsh A, Mancini GB. Carotid intima-media thickness measurements: what defines an abnormality? A systematic review. Clin Invest Med 1999;22:149-57. 